Prophet die Wirkung seiner Drohrede durch einen prosaischen, in der symbolischen Handlung durch nichts angedeuteten ${ }^{1}$ Zusatz abschwächt. Gehörte der v. s zum ursprünglichen Bestande, so müßte man nach v. 3 die ausdrückliche Zusicherung erwarten, dab nach der Zeit der Buße die Restitution in die Rechte der Ehefrau eintreten werde. Dann hätten wir aber wieder an Stelle einer einzelnen auferordentlich drastischen Pointe einen Vergleich zwischen zwei Reihen, bei denen die alten Schwierigkeiten wieder aufleben, da der Abfall des Volkes von Gott $\mathrm{n}$ ach der Erwählung nicht der Buhlerei der Frau vor der Ehe mit Hosea entspricht. Dagegen läßt sich v. $s$ als Interpolation sehr gut begreifen: die Drohweissagung muß in einen versöhnlichen Heilsspruch ausklingen; v. $s$ ist also eine genaue Parallele $\mathrm{zu}$ den allgemein als Interpolation ausgeschiedenen Versen 2 1-4.

Es bleibt jetzt noch die Frage $z u$ beantworten, wie sich Kap. 3 zu Kap. I verhält. Hat Hosea die Dirne des 3. Kapitels, etwa eine Hierodule, später entlassen, nachdem sie ihren Zweck erfüllt hatte, oder hat er sie doch geheiratet, und liegt in $\mathrm{x}_{2}$ eine Anspielung auf ihren ehemaligen Beruf vor? Oder besteht zwischen Kap. I und Kap. 3 überhaupt keine Beziehung? Soviel ich sehe, läßt sich diese Frage vorläufig nicht entscheiden; vielleicht, wird eine genauere Kenntnis des Hierodulenwesens einmal ein besseres Verständnis der Verse I $_{2} 3$ ermöglichen. Vorläufig müssen wir uns damit begnügen, daß in $3_{1-4}$ ein in sich abgeschlossener und aus sich selbst wohl verständlicher Bericht über eine symbolische Handlung des Propheten vorliegt.

[Abgeschlossen den 16. Februar 1922.]

\title{
Die erste Elifaz-Rede Hiob Kap. 4 und 5.
}

\section{Von Lic. theol. Eva Gillischewski in Königsberg in $\mathrm{Pr}$.}

Über die Frage, welche Verse in Kap. 4 und 5 des Hiobbuches der ersten Elifaz-Rede ursprünglich angehören und welche als spätere Zusätze zu beurteilen seien, gehen die Ansichten der Ausleger nicht unbeträchtlich auseinander.

1 יכוים רבים heißt nur "lange Zeit“ und besagt, daß die Strafe der Dirne -wie die des Volkes lange dauern soll, nicht schnell vorübergeht. 
In Kap. 4 werden v. 8 f. von DuHM im Kommentar ${ }^{1}$ I 897 stark angezweifelt, in der Übersetzung 1907 ausgeschieden; v. sof, werden nur von SteUerNagel bei KAUTzSCH igio, BUDde ${ }^{2}$ igi3, Volz I92 I beibehalten; die meisten, WELLHAUSEN I871, SIEGFRIED I893, DELITZSCH 1902, DUHM und TORCZYNER 1920 entfernen diese Verse. Neuerdings wird der ganze Visionspassus von TORCZYNER als ein Fremdkorper in unserer Rede bezeichnet. Sehr stark ist auch der Verdacht gegen v. 6 f. in Kap. 5. Hier ist es nur DELITZSCH, STEUERNAGEL, BUDDE, die für ihre Echtheit eintreten. Neben diesen Stellen liegen noch Zweifel an der ursprünglichen Zugehörigkeit vor gegen einige Einzelverse, nämlich 425110222527 .

Wir wollen im folgenden zunächst zu diesen Fragen Stellung nehmen und dann unsererseits den Gedankengang der Rede aufzuzeigen uns bemühen.

In Kap. 4 wird v. 2, dessen erste Hälfte sehr schwierig ist, nur von BICKELL 1892, entfernt, von allen anderen aber beibehalten. Bemerkenswert scheint $\bar{t}$ mir, daß keine weitere Rede des Elifaz sowohl, wie der beiden anderen Freunde mit einer solchen Selbstbetrachtung eingeleitet wird; auch wird in verschiedenen Übersetzungen, wie der von DeLITzSCH und Volz, der Vers separiert anders DuHM, der ihn in Kommentar und Übersetzung mit v. 3 verbindet. Wie LöHR, BAUdISSIN-Festschrift, S. 308 meines Erachtens nachgewiesen hat, steht dieser Vers ebenso wie $5_{27}$ außerhalb des strophischen Systems. So auch P. VETTER nach D. H. MÜLleR, XIV. Jahresber. d. israel.-theol. Lehranstalt in Wien, 1907, S. 82 A I, vgl. LOHR a. a. O. Die Sonderstellung, die dem Verse durch seinen Inhalt wie auch in formaler Hinsicht angewiesen wird, läßt einen Verdacht gegen seine Echtheit zum mindesten sehr begründet erscheinen. - DuHMs Bedenken gegen. 48 t. sind in erster Linie formaler Natur; er bezeichnet diese Verse als "holprig gebaut" und „unsymmetrisch“; außerdem erscheint es ihm „unklar, was sie im Zusammenhang sagen wollen", sind sie „eine Lehre, ein Trost oder eine Warnung für Hiob"? - Es scheint mir deutlich, daß die beiden Sätze, über deren stilistische Beschaffenheit ich nicht urteilen will, das Pendant sind zu v. 6 und besonders v. 7. Der letztere soll durch

$1 \mathrm{Da}$ die einschlagige Literatur in Fachkreisen hinreichend bekannt.ist, ist von einer bibliographisch genauen Angabe derselben des Raumes wegen abgesehen. 
seine Frage Hiob nötigen, einzuräumen, daß ein Unschuldiger nicht zugrunde geht, und $\mathrm{zu}$ diesem Gedanken stellt Elifaz ergänzend seine Erfahrung, daß nur Böse zugrunde gehen. Für Hiob sind diese Worte v. 1-9 - diesen Gesichtspunkt gibt v. 6 klar an die Hand - eine tröstliche Stärkung. Daß 410 f. zwei Sprichwörter sind, die den Zusammenhang zwischen dem Geschick des Bösen und dem der Seinen, nicht, wie der Kontext fordert, dás persönliche Geschick des Bösen behandeln, darauf hat LOHR zur Genüge hingewiesen. Gerade dadurch charakterisieren sich diese Verse als Beischriften, als welche sie auch von der Mehrzahl angesehen werden. Wenn BUDDE ihre Streichung als „Willkür" bezeichnet, so dürfte sich dieses Urteil mit mehr Recht auf Volzs Übersetzung anwenden lassen, die mit Umstellung von v. rob und v, xrb so lautet:

Der Löwe brillt mit wilder Stimme und seine Jungen streifen stolz (') -

$\mathrm{Da}$ muß er elend Hungers sterben den Jungleun bricht man das Gebiß! -

Gegen die Echtheit der Traumvision hat neuerdings ToRCZYNER Bedenken erhoben, S. I 2 ff. Da .v. s ff. mit dem Gedanken vom Untergang der Sünder zum Inhalt von v. 8 f. - zurückkehre, so sei die Traumvision, daß Engel und Menschen vor Gott niemals rein erscheinen können, eine Unterbrechung. Zu dieser Annahme zwingt nach TORCZYNER S. 13 vor allem auch der Umstand, daß ja durch die Tatsache, daß kein Mensch vor Gott gerecht sei, dem Gerechten die Anerkennung seiner Gerechtigkeit und der Trost daraus entzogen würde. Diese Ụ̂berlegung führt TORCzYNER zu der Vermutung, daß der ganze Visionspassus einer Hiobrede entstamme. Um zu TORCZYNERs Gedanken die richtige Stellung zu gewinnen, ist hervorzuheben, daß Elifaz in dem Visionspassus von der nicht vorhandenen absoluten Reinheit des Menschen Gott gegenüber handelt im Unterschiede von der relativen Reinheit gewisser Menschen gegenüber anderen. Durch diesen Passus will Elifaz einer falschen Selbstbeurteilung in.Hiob vorbeugen. Und wenn er von der sittlichen Mangelhaftigkeit der Menschen im Blick auf den allein Reinen v. $x$ übergeht $z u$ ihrer physischen Hinfälligkeit v. 20 f. und dann sich speziell wieder zưr Vergänglichkeit der Bösen wendet $5_{2}$ ff , so kehrt er damit allerdings $z \mathrm{u}$ dem Gedanken von 48 f. zurück, aber sein Exkurs ist wohlbedacht und darf als nichts weniger denǹ als eine 
störende „Unterbrechung“ empfunden werden. - $5_{1}$ wird von BUdde, SIEUERNAGel, Volz als echt angesehen, von SIEgFried, BeER, DuHm, LOHR, TORCZYNer, wie mir scheint, mit Recht gestrichen. Es unterbricht jedenfalls diese Apostrophe an Hiob die Schilderung $4_{21} 5_{2}$. Ob es, wie DuHM meint, eine Randbemerkung zu 4 is sei, lasse ich dahingestellt. Die Ausscheidung von 56 f. schon seitens Wellhausens, dann Duhms, Richters, Volzs, - TORCZyNER S. I6 f. nimmt, um die Verse zu halten, eine Lücke im Vorhergehenden an - wird vorwiegend von DUHM begründet; die andern schließen sich seiner Argumentation an. DuHM zitiert v. 6 und fragt: „Warum wird dies in Abrede gestellt? Hiob hat es jedenfalls nicht behauptet." - Demgégenüber ist zunächst mit Nachdruck darauf hinzuweisen, daß die Rede des Elifaz doch überhaupt nur eine ganz allgemeine Beziehung zum Monolog des Hiob hat. Des letzteren Klage darüber, daß man ihn in dieses traurige Leben hineingestoßen, beantwortet Elifaz mit einem Hinweis auf die nach seiner Meinung auf dem Vergeltungsdogma ruhende göttliche Weltordnung. Will man aber spezielle Beziehungen zwischen beiden suchen, so läßt sich Hiobs Gedanke, daß dieses irdische Dasein voll ist an Mühen, Leiden, Ärger usw. $3{ }_{17}$ ff. sehr gut mit des Elifaz' Worten 56 f. zusammenstellen; denn sie wollen doch nichts anderes sagen, als was unser Dichter so ausdrückt: die Welt ist vollkommen überall, wo der Mensch nicht hinkommt mit seiner Qual. - Zu der Streichung von $5_{10}$ kann hier auf LÖHR S. 309 verwiesen werden; DELITZSCH und SteuERNAGEL behalten den Vers bei. Ebendort vgl. über v. 22 25 27. Der erstere wird nur von DuHM und Volz entfernt; alle drei von BuDDE, DELITZSCH, STEUERNAGEL beibehalten. Während TORCZYNER v. ${ }_{22}$ und 25 beibehält, ist er gegen v. ${ }_{27}$ skeptisch. Besonders v. 27 sticht inhaltlich gegen die milden, versöhnlichen und abschließenden Worte von v. ${ }^{26}$ stark ab.

Bevor wir nun den Gedankengang der Rede aufzeigen, geben wir sie in deutscher Übersetzung wieder in der Gestalt, die wir als die ursprüngliche glauben bezeichnen zu dürfen.

4 3 a Sieh du hast viele vermahnt, 3 b hast schlaffe Hände gestärkt 5 Jetzt . . . . . . . wie's dich traf, wardst du verdrossen,
4 a den Gefallenen mit Worten aufgerichtet, $4 \mathrm{~b}$ und wankende Kniee gefestigt ${ }^{1}$.

da's dich faßte, warst du entsetzt.

\& Vgl. Jes 35 . 
6 Ist dir nicht dein Glaube eine Stütze, gibt Zuversicht dir nicht dein Wandel?

7 Denk doch, wer ging unschuldig zugrunde, und wo wurden Gerechte vernichtet?

8 Soviel ich weiß, sind's, die Unheil gepflügt, die Böses gesäet, die es ernten.

9 Vom Hauche des Höchsten vergehn sie, durch sein Zorneswehn nehmen sie ein Ende.

12 Zu mir stahl sich ein Wort, es vernahm mein Ohr ein Geflüster,

13 a beangstigt von nächtlichen Gesichten ${ }^{1}, \quad 14$ a da Schreck mich erfabte und Zittern.

$14 \mathrm{~b}$ Schier in allen meinen Gliedern er- $15 \mathrm{~b}$ es strabte sich mir das Haar am bebt' ich, Leibe.

15a Ein Geist $20 g$ an meinem Gesicht voruber $16 \mathrm{a}$ und verbante vor meinen Augen, 16 a doch nicht erkannt ich sein Aussehen, $16 \mathrm{~b}$ nur hört ich ein leises Säuseln:

17 „Ist ein Mensch vor Gotte gerecht oder fehllos vor seinem Schöpfer ein Mann? 18 Sieh seinen Dienern traut er nicht und Irrtum legt er seinen Engeln bei.

19 Geschweige daß die Bewohner von Lehmhäusern als rein sollten gelten vor ihrem Schopfer" ?

20 a Im Umsehn sind sie vernichtet, $20 \mathrm{~b}$ unbeachtet vergehn sie für immer.

21 Nicht wahr? - Ist herausgerissen ihr Zeltpflock, so sterben sie, und nicht in

Weisheit ${ }^{3}$.

52 Den Toren bringt um seine Unzufriedenheit, dem Narren gibt seine Unrast den Tod 4 ,

4 bar werden seine Kinder jeder Hilfe, jedweden Rechtes rettungslos beraubt ${ }^{5}$.

3 Ich hab gesehn den Toren Wurzel schlagen, doch entwurzelt ${ }^{\circ}$ war seine Stätte

gar plötzlich,

5 uber seine Einte fiel her der Hungrige, es schöpften Lechzende von seiner Milch ? 6 Nicht aus dem Boden steigt das Unheil auf und aus dem Acker nicht wächst

7 der Mensch allein erzeugt das Ungemach . . . . . . . . . das Ungemach,

8 Doch ich würde mich wenden an Gott und dem Höchsten vertraun meine Sache, 9 der Großes vollbringt unerforschlich und .Wünder wirkt ohne Zahl,

I I der Niedrige zur Höhe fürt und Trauernde durch Glück erquickt,

I 2 der die Gedanken der Klugen vereitelt,. daß sie nichts Verständiges vollbringen,

13 der die Schlauen mit ihrer,eignen Klugheit fängt, daß ihr listiger Plan sich übersturzt, 14 sie am hellen Tag ins Dunkel geraten und wie im Finstern tappen zur Mittagszeit. I5 Doch rettet er vor dem Schwert den Armen ${ }^{8}$ und aus der Gewalt des Tyrannen den Schwachen,

16 so daß dem Geringen eine Hoffnung erwächst, der Frevler aber verstummen muß.

$1 \mathrm{Zu}$ der Umstellung der Versteile vgl. ToRCzynER, S. Io ff. - In Anlehnung an seine Ausführungen ist oben übersetzt. Standen im Archetypus die Halbverse unter- statt nebeneinander? .

$0^{2}$ Vgl. Löhr, S. 3 ro. $\quad{ }^{8}$ Im Gegensatz zu LöHR, S. 3 II.

4 Als Inhalt von ka'as und qin'â sehe ich das.Streben des Toren nach Besitz und Reichtum an, vgl. 5 .

${ }^{5}$ Stellung dieses Verses abweichend von LöHri, S. 3 II.

- In Löhrs Konjektur sehe ich nur einen Versuch, der Stelle einen kontextgemäßen Sinn zu geben. 'In' Anlehnung an TORCzyner u. a:

8 Mit TorCzyner u. a. 
I7 Wohl dem, den der Höchste zurechtweist, der des Allmächtigen Züchtigung nicht verschmäht;

I 8 denn er ist's, der verwundet und verbindet, und ob er schlägt, er heilt doch auch.

I9 Aus sechs Gefahren reißt er dich heraus, und in der siebenten trifft dich nicht das Ungluck.

20 In Hungersnot erlöst er dich vom Tode und in der Schlacht aus der Gewalt des Schwertes.

2I Vor der Zunge des Verleumders bist du bewahrt, brauchst nicht zu fürchten das nahende Unheil.

23 Mit den Felddämonen stehst du im Bunde, hast Frieden mit den wilden Tieren.

$24 \mathrm{Du}$ weißt, daß sicher steht dein Zelt und der Ertrag deines Ackers bleibt nicht aus; 26 im Alter gelangst du ins Grab wie die Ernte zu ihrer Zeit auf die Tenne.

Der Gedankengang ist demgemäß folgender:

Elifaz beginnt und schließt seine Rede mit einer Bezugnahme auf die Person Hiobs. Dessen Schrei nach Erlösung von einem Leben, das ihm nur als Qual erscheint, setzt er, Elifaz, im allgemeinen den Gedanken einer gerechten, göttlichen Weltordnung entgegen, die auf dem Satz ruht: der Gute wird belohnt, nur der

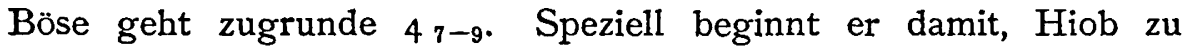
mahnen, daß er, da ihn das Unglück erfaßt, nicht gleich verzweifeln dürfe, 45 , sondern sich des Standpunktes erinnern müsse, den er selbst früher gegenüber dem Unglück anderer eingenommen. Übrigens falls er, Hiob, sich Gott gegenüber in besonders günstiger Position wähnt, d. h. wenn er sich für einen absolut reinen Menschen hält, so möge er wissen, daß es solche überhaupt nicht gibt. Elifaz weiB das aus Offenbarung in Form einer nächtlichen Vision und Audition. Schon hier zeigt sich, was auch im folgenden hervortreten wird, daß Elifazs Worte stets eine ganz persönliche Abzweckung haben. Die sittliche Schwachheit der Menschen führt den Redner auf ihre körperliche Hinfälligkeit; sie sterben, und zwar unweise 421 . Den einen tötet kacas, den andern qin'â. Diese Begriffe sind $z u$ verstehen von dem rastlosen Streben nach Besitz, und zwar, dem Milieu gemäß, dem des Landmannes, vgl. $5_{5}$. Solch Tor geht zugrunde; seine Familie mit ihm $54^{1}$, und sein Besitz fällt anderen zu 5 5. Nicht sein Grund und Boden'ließ und läßt sein Verhängnis hervorsprossen, sondern -er selbst war und ist der Urheber seines Verderbens $5{ }_{6}$ f.' $^{\prime}$ Auch diese Verse enthalten, natürlich versteckt, eine' sehr direkte Spitze gegen Hiob. Es wird ihm

1 Sind die Vèrse $4_{10}$ f. vielleicht eine in die falsche Kolumne geratene Glosse zu 5 ? 
hier recht verständlich bedeutet, in sich selbst die Ursache seines Unglücks zu suchen. Nach dieser doppelten Mahnung, nämlich sich

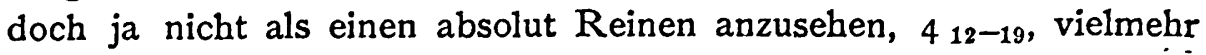
bei sich selbst den Grund des Unglücks $z u$ suchen 420 \&. $5{ }_{2-7}$, kommt ein positiver Rat $58 \mathrm{ff}$ : ich würde mich, an deiner Stelle nämlich, an Gott wenden, dessen Wesen, wie jetzt 5 11-16 beschreiben, in der ausgleichenden Gerechtigkeit gipfelt. Man beachte dabei noch, wie künstlich dieser Passus disponiert ist: v. $x$ die armen Frommen, v. ${ }^{12}$ die stolzen Bösen; v. $x_{3}$ f. ist von den letzteren, v. $x_{5}$ f. von den ersteren die Rede. Das Thema von diesen Frommen, die von gottgesandtem Unglück heimgesucht werden, gibt den Übergang zum letzten Passus $5_{17-26}$, der wieder ganz persönlich ist, insofern als er in 517 erstens die Mahnung enthält $\mathrm{zu}$ geduldigem Ausharren und zweitens durch Aufzählung von sechs Bedrängnissen den Hiob sicher an persönliche Lebenserfahrungen erinnern soll. Denn man wird doch wohl mit größerer Wahrscheinlichkeit annehmen dürfen, daß diese Aufzählung nicht rein theoretisch ist, sondern daß sie auf Erlebnisse Hiobs anspielt; nur das gewährleistet doch eine rechte Wirkung auf letzteren; und endlich drittens durch den Ausblick auf ein friedsames normales Lebensende ganz besonders dem stürmischen Verlangen nach dem Tode seitens Hiobs in Kap. 3 entgegentritt.

[Abgeschlossen den 14. Januar 1922.]

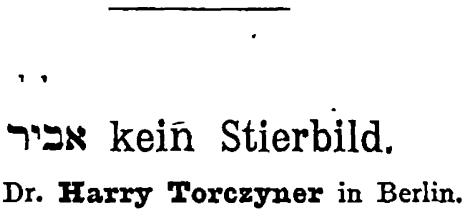

Im Jahrg. XXXIX Heft I dies. Zeitschr., S. 38 trägt K. BuDDE die These vor, אפור sei an mehreren Stellen der Bibel ein später Ersatz für אביר; dieses letztere bezeichne ein Stierbild. Da nun auch H. GRESSMANN in seiner jüngst erschienenen Schrift über die Lade Jahwes aus dem Beinamen der Gottheit אביר יעקב ähnliche Schlüsse zieht, liegt die Gefahr nahe, daß die Fabel vom Stierbild -Ephod weitere Kreise ziehe. Darum teile ich hier aus einer an anderer Stelle ${ }^{1}$ erscheinenden Abhandlung über die Bundeslade und

1 Festschrift der Hochschule für die Wissenschaft des Judentums, Berlin 1922. 